\title{
RELIABILITY AND SURVIVAL ANALYSES OF MOBILE OS VIA WEIBULL MODEL
}

\section{JULLIARD RALIHALIZARA, PH.D ${ }^{1} \&$ JULES HARRIS DANARSON, PH.D ${ }^{2}$}

${ }^{1}$ School of Management, Ecole Supérieure des Sciences Agronomiques, Antananarivo University,Madagascar

${ }^{2}$ School of International Education, Guangxi University of Finance and Economics, Nanning, Guangxi,P.R. China

\begin{abstract}
Mobile devices have become a crucial part of our daily life; we rely on those gadgets to carry sensitive personal and corporate data. Having a secure and up-to-date device not only conveys trust and peace of mind to the end-user but also brings real-time protection against security threats and vulnerabilities. This paper brings an objective study, inspired by the questions of how security patches and updates reach end-users and what we can learn from the current pattern of how they are delivered. In addition, it focuses on the healthiness of the main operating systems, which govern mobile devices, namely Android and iOS, by using the theoretical framework of reliability and survival analyses via Weibull model. Survival models built from the life data of the two main mobile operating systems are subsequently validated by Anderson-Darling hypothesis tests, and the best fits are chosen from the Root Mean Square error criteria. The obtained results give some indication of the pattern in which security updates and patches reach the end-user. A final, reliability analysis of life data allows getting to the insight of the evolution of the hazard rate and the related reliability function, for both mobile operating systems through graphical and numerical results.
\end{abstract}

KEYWORDS: Mobile operating System, Reliability Analysis, Survival Analysis

Received: Dec 04, 2020; Accepted: Dec 24, 2020; Published: Jan 28, 2021; Paper Id.: IJISMRDDEC20202

\section{INTRODUCTION}

Mobile devices have become deeply involved in our daily life in today's modern society, and they seem to be here to stay. Besides, the very practical mobility feature, the increasing computing power and the multitude of applications available, rendered the traditional use of "feature phone" obsolete. Modern smartphones now offer a lot more than the traditional telephony functionality. As a matter of fact, mobile devices now serve as camera, as map and Global Positioning System receiver, wallet for electronic money and recently, as health monitor [1], or a health status check and recorder as seen in China (and probably in other countries) following the outbreak of COVID-19, etc. Moreover, the number of mobile devices users are not ceasing to grow. Some developing parts of the world are even currently experiencing a technology leapfrog. Meaning that, those countries are skipping the old fixed phone technology of the previous century, to directly land to the use of modern mobile phone of the 21 st century. Their first exposure to modern communication and modern computing is through mobile devices, and this, without prior knowledge of the preceding technology, without even knowing about computer basics and how communication works. Circumstances also force us to get engrossed with mobile phones. In Nanning, like many cities in China, you cannot go out and roam around during this pandemic period without your mobile phone showing a code of green color appearing on its screen, meaning you are coronavirus symptom-free or healthy as opposed to yellow for a suspected case or red being in need of a long quarantine or treatment. Thus, a mobile phone has 
recently become a crucial part of our physical being.

Furthermore, mobile devices have not ceased to evolve since the launch of the first iPhone by Apple in 2007 [2]. Early versions of the gadgets are usually shipped to the end-user with bugs and flaws. And bug fixes, patches, along with functionality enhancements and upgrades are often available to the end-user gradually, in a form of update packages. Thus, in order to keep the personal and corporate data secure, responsibilities are shared between the end-users and manufacturers. This requires a rigorous development standard from the operating system (OS) manufacturers, but also a minimum level of security awareness from the end-users' side. Under such circumstance, and given the relatively short story of smartphones, the following questions are raised: firstly, how do security updates and patches reach the end-users? Secondly, is it possible to sort out a pattern about the current state of mobile operating system release and update pattern? And if so, what can be concluded?

At first glance, the above-given questions seem trivial, since mobile OS are pushed out by companies and organizations, which surely have their own planned release schedules and marketing strategies. Thinking outside the box, however, bug discoveries, followed by the related bug-fixes and then patch delivery seem random. In addition, security updates are not likely to reach the end-user on a regular basis. To get a better understanding of the underlying process, the main focus is put on the main operating system of mobile devices. The analysis is made from the theoretical perspective of reliability and survival analysis, through the use of the Weibull probability distribution.

This paper starts with a literature review, followed by a methodology, laying down the theoretical ground for the main inquiry. Models are built for life data taken from the two main mobile operating systems: Android and iOS. The obtained Weibull life models are subsequently validated via Anderson-Darling hypothesis tests. And the best fit is chosen from the Root Mean Square Error criteria. The results show that Android life data can be assimilated to a Weibull model as: W $(91.7276,0.9644)$, while the iOS life pattern can be expressed as W $(39.2413,1.2199)$. A reliability analysis exhibits a constant hazard rate through time for the Android model while the latter is increasing for the iOS mobile operating system. This study ends up with a discussion of the result followed by some concluding remarks.

\section{LITERATURE REVIEW}

Android currently detains $86.1 \%$ of the global market, while apple detains $13.7 \%$ [2]. Together, they account for $99.8 \%$ of the mobile devices currently circulating in the world. Android and IOS mobile operating systems are developed and sustained under two different business and technical environments. The Android mobile OS, defined on its own website as an "open-source operating system for mobile devices and a corresponding open-source project led by Google" [3]. The open-source license allows android source code to be accessible by various designers, developers and tech hobbyists. Android mobile OS can be installed in almost any device, the source code is available for customization and the documentation is publicly available. Android has a decentralized policy with regard to development and, in term of vulnerability, Pavneet et al. [4] reported that most Android OS bug discoveries and reports are posted by common endusers whereas highly skilled developers mainly report only in the field of more technical and more specific projects. Besides, the formerly called iPhone OS and currently named iOS is a proprietary mobile OS owned by Apple. It only runs on Apple hardware. Contrary to the open-source and crowd-sourced Android development environment, Apple development process is classified, as is often referred to, as a "walled garden," a closed environment under the sole control of Apple [5], [6]. This applies to both operating system and application development. 
Regarding security issues, the publicly available database for Common Vulnerability and Exposure (CVE) maintained by Mitre Corporation gives an understanding about the vulnerabilities affecting various systems. As for 2019, a total of 156 vulnerabilities have been disclosed for Apple iOS while 414 vulnerabilities have been reported on Google Android [7]. Among the recorded vulnerabilities are: information leak, code execution, etc. A study on bug reports affecting third-party libraries and bug reports affecting the core Android OS in [4] has showed that bugs affecting third party libraries also have a strong correlation with the bugs weakening the main OS. The survivability aspect of the Android operating system was addressed by Vasquez et al. [8] while tackling with Android-related vulnerabilities. Some authors pointed out that third-party hardware driver manufacturers are among the main vector of vulnerabilities embedded into the OS. More alarmingly, such vulnerabilities can sometimes remain unnoticed for a long period of time. As a matter of fact, a newly introduced vulnerability can stay dormant in the OS for a very long period before being identified and fixed, leaving the end-user exposed to various security risks. And even if a vulnerability fixes are available, Erik Deer et al. [9] stressed out that the time-lapse between the discovery of a security flaw and the time fixes reaching the user is "desperately long and unacceptable." In some cases, security patches never reach the end-user at all. In 2017, Thomas et al. [10] sorted out that, on average, an Android user gets only around 1.26 updates per year for their devices. Finally, comparative studies about the two mobile OS made by Kumar et al. in [2] and Patel et al. in [11] argued that, compared to Android and given the development environment and the close control Apple has upon the development process and application ecosystem, iOS is slightly more secure and less likely to be the target of attacks.

As mentioned in the introduction, security concern is not the sole responsibility of the manufacturers; smartphone users also have their own share of it in order to keep themselves safe. In an attempt to educate the user, the Protection Motivation Theory (PMT) has been largely used in order to find the most appropriate security-aware behaviors. For example, by introducing the notion of an anticipated regret to the PMT, Silas [12] has demonstrated that an anticipated regret has a positive correlation with the preventive action taken by the users. For the same purpose, Bartlomie et al. [13] made a distinction between "perceived vulnerability" and "perceived severity," and pointed out that both can trigger a security-awareness behavior. Some authors also stressed on the role of the "emotional arousal" caused by the perceived seriousness of the vulnerability, which acts as a threat and a motive for the users to be more prudent. Furthermore, Alsaleh et al. in [14] found out that one security practice can lead to another one. As it happens, through PMT and threat Avoidance Theory, several authors demonstrated the correlation among different security-related behaviors such as: device locking, applying security patches, etc. During the decade-long history of mobile devices however, Murat et al. in [15] assessed that the current level of awareness among mobile phone users is still far from a satisfactory level, and needs to be improved to deal with the modern and complex devices. Some authors also singled out cybersecurity training as the most important factor which affects the users' behavior toward safety, while other factors such as age and education level matter less.

Although responsibilities are shared between users and manufacturers, this study focuses on the share of the accountabilities the OS developers and maintainers have only. Leaving aside the approach, which aims to adjust the users' behavior towards technology, this study assumes that having a secure and stable main OS suffice for the users to feel safe and be genuinely safe is crucially important. As a matter of fact, this should be the starting point and the minimum effort required to maintain the safety of the circulating devices. Therefore, this study is disregarding any other advanced usage and their security implication, such as application handling and network usage. End-users need only to keep up with the latest version of the OS, which is presumably the most secure, the most stable and most advanced. The only concerns users have is to know if patches and fixes are available with regard to the latest known vulnerabilities and, if those fixes are 
available for them to secure their devices. Although bug fixes and security patches are not the only reason for OS manufacturers to push out a new version since a new version can be released in order to deploy new features or enhance the old ones. Nevertheless, for this case, we are assuming that being upgradeable renders an OS obsolete. Furthermore, Mobile OS life data are taken as they are, from an outside point of view, without considering any planned strategy or policy from the manufacturer.

Furthermore, this paper aims to raise further questions in the area of technological planning and human behavior toward technology, especially toward alarms and notifications. For example, Eisenberger et al. in [18] have brought an insightful remark on how human alarm system works, and found out that it works in two steps: a discrepancy monitoring step and a sounding step. The first step aims to detect deviations from a desired standard followed by a second step which signals that a problem has occurred and needed to be addressed. Similarly, regarding human reaction and fair process effect by Kees in [19], an extension of this work can be formulated as: is the current pattern of mobile OS update delivery enough to give raise to personal uncertainty and insecurity in order to activate the human alarm system and compel the end-users to take action? And how are those notifications being perceived by the users?

Before going any further, it is worthwhile to focus on the term 'obsolete', itself. The dictionary definition of obsolete gives: "not in use anymore, having been replaced by something newer and better or more fashionable." In the area of technological products, obsolescence was discussed by Cooper et al. in [16] and Volker G. et al. in [17], and it is said to occur when products go "out of use" or "out of date." Cooper et al. made a clear distinction between two sorts of obsolescence: "obsolescence of quality" and "obsolescence of desirability." The first happens when products break down or wear out at a given time, while the second type of obsolescence, also called "psychological obsolescence," takes place when a product is still acceptable in terms of quality and performance, but its subjectively is considered "worn out" in the mind of the users. The latter happens because a styling or other changes have made the product seem less desirable in their eyes. For this work, the term obsolete is referring to its first meaning i.e. obsolescence of quality as defined above. In other words, "an existing product becomes obsolete, when a product is introduced, that performs the function better" [16]. For the subsequent life data consideration, it is being assumed that a given OS version is taken as obsolete when a newer version is publicly made available. A more recent version of a software usually comes up with fixes and enhancements, and performs better than the previous one. Hence, the life span of a given OS version is the computed period between its date of release and the release date of the following version.

To conduct the reliability and survival analysis of mobile operating systems, the following section of the paper focuses on the building of a reliability and survival model of the Android and iOS operating systems. With the use of the two-parameter Weibull distribution, the obtained results will be the topic of the subsequent discussion in terms of survival analysis.

\section{METHODOLOGY}

\section{The Two-Parameters Weibull Distribution}

The Weibull distribution, set up by the Swedish engineer Waloddi Weibull in 1939, is herein used to model the life data of mobile OS. Initially applied in material science, the Weibull distribution is now widely used in various fields such as engineering, physics, chemistry, meteorology, medicine, and economics [20]. The two-parameter Weibull distribution is by far the most widely used distribution for life data since it adequately describes the observed failure times of different types 
of components and phenomena [21], [22]. The probability density function (PDF) of a two-parameter Weibull distribution $\mathrm{W}(\alpha, \beta)$ is defined as [23], [24]:

$$
f(x)=\frac{\beta}{\alpha}\left(\frac{x}{\alpha}\right)^{\beta-1} e^{-\left(\frac{x}{\alpha}\right)^{\beta}} x>0,(\alpha, \beta)>0
$$

Where $\alpha$ is the scale parameter and $\beta$ the shape parameter and, $\mathrm{x}$ the time to failure. The corresponding Cumulative Distribution Function (CDF) is given by [22]:

$$
F(x)=1-e^{-\left(\frac{x}{\alpha}\right)^{\beta}}
$$

\section{Parameters Estimation}

Three main techniques are going to be used to estimate the scale and the shape parameter of the Weibull model: the moment method, the graphical method and the maximum likelihood method.

\section{The Moment Method}

The mean and the variance of the Weibull distribution are respectively written as [23], [25], [26]:

$$
\begin{gathered}
\mu=\alpha \Gamma\left(1+\frac{1}{\beta}\right) \\
\sigma^{2}=\alpha^{2} \Gamma\left(1+\frac{2}{\beta}\right)-\mu^{2}
\end{gathered}
$$

From equation 3 and equation 4, the following can be deduced:

$$
\ln (\mu)=\ln (\alpha)+\ln \Gamma\left(1+\frac{1}{\beta}\right)
$$

And, $\ln \left(\sigma^{2}+\mu^{2}\right)=2 \ln \alpha+\ln \Gamma\left(1+\frac{2}{\beta}\right)$

Equation 5 and equation 6 can be reduced into:

$$
\ln \Gamma\left(1+\frac{2}{\beta}\right)-2 \ln \Gamma\left(1+\frac{1}{\beta}\right)-\ln \left(\sigma^{2}+\mu^{2}\right)+2 \ln \mu=0
$$

From the solution of equation $7, \alpha$ can be retrieved following equation 8 :

$$
\alpha=\frac{\mu}{\Gamma\left(1+\frac{1}{\beta}\right)}
$$

\section{The Graphical Estimation Method}

Equation 2 can be expressed in the form of a linear equation $y=\beta x+b$ as:

$$
\ln (-\ln (1-F(x)))=\beta \ln (x)-\beta \ln (\alpha)
$$


With $\mathrm{n}$ being the size of the sample and $\mathrm{F}$ being the empirical distribution function, $\mathrm{F}$ was computed in various ways according to the plotting positions listed below [20], [26].

Table 1: Plotting positions for the graphical estimation method

\begin{tabular}{|l|c|}
\hline \multicolumn{1}{|c|}{ Plotting position } & $F$ \\
\hline Naive Estimator or maximum likelihood estimator & $F=\frac{i}{n}$ \\
\hline Weibull or Mean position & $F=\frac{i}{(n+1)}$ \\
\hline Midpoint position or Median Rank estimator & $F=\frac{(i-0.5)}{n}$ \\
\hline BLOM position & $F=\frac{(i-0.375)}{(n+0.25)}$ \\
\hline Median position & $F=\frac{(i-0.3)}{(n+0.4)}$ \\
\hline Mode position & $F=\frac{(i-1)}{(n-1)}$ \\
\hline
\end{tabular}

From the equations above, the main principle of the graphical estimation method is to find the best straight line to fit the linear model of equation 9. The gradient of the line gives the value of $\beta$ and the corresponding $\alpha$.

\section{The Maximum Likelihood Estimation}

The maximum likelihood estimation (MLE) method is defined by taking the unknown variables and computing the joint density of all observations of the data set, which is assumed to be independent and identically distributed. The maximum of the likelihood function is found once it is defined. From the expression of the PDF of the Weibull distribution in equation 1, this method consists in finding the values of beta and alpha, which maximizes the "likelihood" of obtaining the observed data [22], [24].

$$
L(\alpha, \beta)=\prod_{i=1}^{n} \frac{\beta}{\alpha}\left(\frac{x_{i}}{\alpha}\right)^{\beta-1} e^{-\left(\frac{x_{i}}{\alpha}\right)^{\beta}}
$$

Maximizing $L(\alpha, \beta)$ is equivalent to the maximization of $\operatorname{Ln} L(\alpha, \beta)$.

$$
n[\ln -\beta \ln \alpha]+(\beta-1) \sum_{i=1}^{n} \ln x_{i}-\sum_{i=1}^{n}\left(\frac{x_{i}}{\alpha}\right)^{\beta}
$$

Matlab computer code was written to help find the optimal value of parameters $(\alpha, \beta)$ and, to give a graphical representation of the couple of solutions.

\section{Anderson-Darling hypothesis test}

Regarding the hypothesis test, the Anderson-Darling (A-D) test is being used. Developed by T.W. Anderson and D.A. Darling in 1952, this method is an alternative to other statistical tests used in order to determine a sample's distribution. It has been argued that, in some cases, the A-D test can be a better option compared to the commonly used KolmogorovSmirnov (K-S) test. In fact, in [27], the K-S test is argued as less powerful than the Anderson-Darling and Cramer-Von Mises test. For this study, the A-D test is retained to assess the validity of the computed model against the collected data. 
Inspired by [26], [28] and the method used in the Military Handbook (MIL-HDBK-17-1F) the A-D hypothesis test for a Weibull distribution can be summarized as:

H0: The samples follow the Weibull law $\mathrm{W}(\alpha, \beta)$ (the null hypothesis).

H1: The samples do not follow the Weibull law.

Considering the Anderson-Darling statistic where $\alpha^{*}$ and $\beta^{*}$ are the estimated parameters values,

$$
\begin{gathered}
z_{(i)}=\left[\frac{x_{(i)}}{\alpha^{*}}\right]^{\beta^{*}} \\
A D=\sum_{i=1}^{n} \frac{2 i-1}{n}\left[\ln \left(1-e^{-Z_{i}}\right)-Z_{n+1-i}\right]-n
\end{gathered}
$$

Where: $\left\{\mathrm{z}_{1}<\ldots<\mathrm{z}_{\mathrm{n}}\right\}$ are the ordered sample data points and $\mathrm{n}$ is the number of data points in the sample. The Observed Significance Level (OSL) is given by:

$$
O S L=\frac{1}{1+e^{-0.10+1.24 \ln \left(A D^{*}\right)+4.48 A D^{*}}}
$$

Where:

$$
A D^{*}=\left(1+\frac{0.2}{\sqrt{n}}\right) A D
$$

If OSL $\leq 0.05$, at a five per cent risk at being in error, the Weibull assumption is rejected, otherwise there is not enough evidence to reject the Weibull model.

\section{Goodness of fit}

For the accuracy of the fit, the standard Root Mean Square Error (RMSE) is used to measure how close to the theoretical Weibull model the collected data is. The best fit corresponds to the closest value of the RMSE to zero. As showed in equation 16, the RMSE uses the observation data $x_{i}$ and the computed data $y_{i}$ and the number of observations $n$ [29].

$$
R M S E=\sqrt{\frac{1}{n} \sum_{i=1}^{n}\left(x_{i}-y_{i}\right)^{2}}
$$

\section{RESULTS}

In reliability analysis, the main variable of interest is how long, for this case in days, a component would function properly before reaching obsolescence. This lapse of time is usually called the survival time or failure time. To study the lifetime of operating systems, it is assumed that the life of an OS starts from its public release date and it ends when the next release is being pushed out to the public, making the previous version obsolete.

From Android version history, 52 inputs have been collected yielding 51 life data. The iOS version history has 129 versions giving 128 life data. It is important to mention that, so far, not one major version of an OS of either type has been released with zero bugs. This means that early versions are always followed by a succeeding versions bringing out 
new improvements and bug-fixes.

This study only takes into account the final and publicly available versions of the OS, and ignores the intermediary versions such as development versions and beta releases versions. Furthermore, the Android tablet-onlyversion from 3.0 to 3.2.6 versions were skipped as well as the Android Wear only 4.4 versions to $4.4 \mathrm{w} .2$. Similarly, for the iOS, the iPad only version from 3.2, to 3.2.2 were skipped as well as the iPhone 4 only version from 4.2.5 to 4.2.10 CDMA. In addition, the iOS version 2.0 was released before the last update of the version 1 came out. Thus, the version 1.1.5 has been ignored for the life computation. The same consideration was made for the version 6.1.5 and 6.1.6 which were released after the release of the version 7 and, the post iOS 13 updates of 12.4.2 to 12.4.6.

\section{Parameters Estimation}

Detailed results from parameters estimation of life data for Android and iOS are presented respectively in Table 2 and Table 3. All OSL are superior to the threshold of 0.05 , showing that there is no sufficient evidence to discard the null hypothesis, which assumes that the collected data are Weibull distributions

Table 2: Parameters estimation and hypothesis test result from Android life data

\begin{tabular}{|c|c|c|c|c|c|c|c|}
\hline \multirow{2}{*}{ Method } & \multirow{2}{*}{ Position } & \multicolumn{2}{|c|}{ Parameters } & \multicolumn{3}{|c|}{ Hypothesis test } & \multirow{2}{*}{ Test result } \\
\hline & & Shape $\beta$ & Scale $\alpha$ & AD & AD* & OSL & \\
\hline Moment & & 1.0416 & 90.6279 & 0.2236 & 0.2299 & 0.7096 & $H_{0}$ accepted \\
\hline \multirow{6}{*}{ Regression } & Naive Estimator & 0.9815 & 87.1794 & 0.2338 & 0.2403 & 0.6881 & $H_{0}$ accepted \\
\hline & Weibull \& Mean & 0.9644 & 91.7276 & 0.2199 & 0.2261 & 0.7173 & $H_{0}$ accepted \\
\hline & \begin{tabular}{|l|} 
Midpoint \\
\end{tabular} & 1.0353 & 90.1670 & 0.2170 & 0.2230 & 0.7234 & $H_{0}$ accepted \\
\hline & Blom & 1.0144 & 90.5955 & 0.2035 & 0.2092 & 0.7508 & $H_{0}$ accepted \\
\hline & Median & 1.0032 & 90.8370 & 0.2011 & 0.2067 & 0.7556 & $H_{0}$ accepted \\
\hline & Mode & 1.0536 & 89.5671 & 0.2392 & 0.2459 & 0.6766 & $H_{0}$ accepted \\
\hline MLE & & 1.0355 & 90.4327 & 0.2172 & 0.2233 & 0.7229 & $H_{0}$ accepted \\
\hline
\end{tabular}

Table 3: Parameters estimation and hypothesis test result from iOS life data

\begin{tabular}{|c|c|c|c|c|c|c|c|}
\hline \multirow{2}{*}{ Method } & \multirow{2}{*}{ Position } & \multicolumn{2}{|c|}{ Parameters } & \multicolumn{3}{|c|}{ Hypothesis test } & \multirow{2}{*}{ Test result } \\
\hline & & Shape $\beta$ & Scale $\alpha$ & AD & $\mathbf{A D}^{*}$ & OSL & \\
\hline Moment & & 1.1664 & 39.6119 & 0.4121 & 0.4194 & 0.3315 & $H_{0}$ accepted \\
\hline \multirow{6}{*}{ Regression } & Naive Estimator & 1.2199 & 39.2413 & 0.4144 & 0.4217 & 0.3276 & $H_{0}$ accepted \\
\hline & Weibull \& Mean & 1.2103 & 39.9276 & 0.4117 & 0.4190 & 0.3322 & $H_{0}$ accepted \\
\hline & Midpoint & 1.2572 & 39.6142 & 0.4912 & 0.4999 & 0.2176 & $H_{0}$ accepted \\
\hline & Blom & 1.2437 & 39.7018 & 0.4579 & 0.4660 & 0.2611 & $H_{0}$ accepted \\
\hline & Median & 1.2363 & 39.7507 & 0.4431 & 0.4510 & 0.2823 & $H_{0}$ accepted \\
\hline & Mode & 1.2670 & 39.5666 & 0.5208 & 0.5300 & 0.1843 & $H_{0}$ accepted \\
\hline MLE & & 1.2022 & 40.0131 & 0.4098 & 0.4171 & 0.3354 & $H_{0}$ accepted \\
\hline
\end{tabular}


From Figure 1 to Figure 6, the graphical representation of the results from the parameters estimation for both Android and iOS life data is given. Figure 1 and Figure 2 refer to the Weibull probability plot. The solid reference line connects the first and third quartiles of the data, which is extended by the dashed line to the end points of the data. The data points appearing along the reference line show a Weibull distribution. Figure 3 and Figure 4 display the numerical solution of the shape parameters obtained from the moment method. Finally, Figure 5 and Figure 6 give a graphical representation of the couple scale and shape parameters obtained by numerical iteration from the Maximum Likelihood Method.

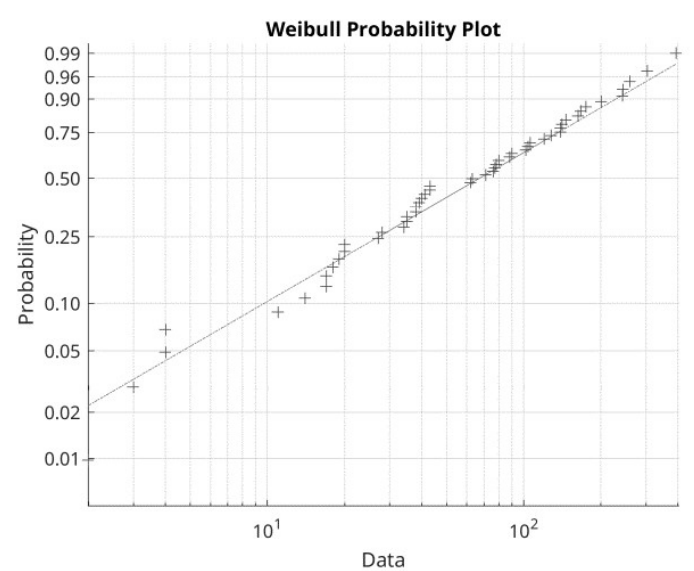

Figure 1: Android Weibull probability plot

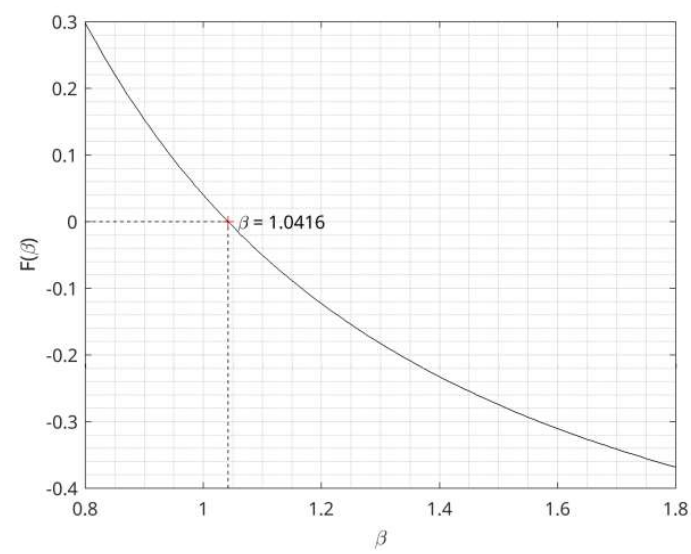

Figure 3: Android Moments Method approximation

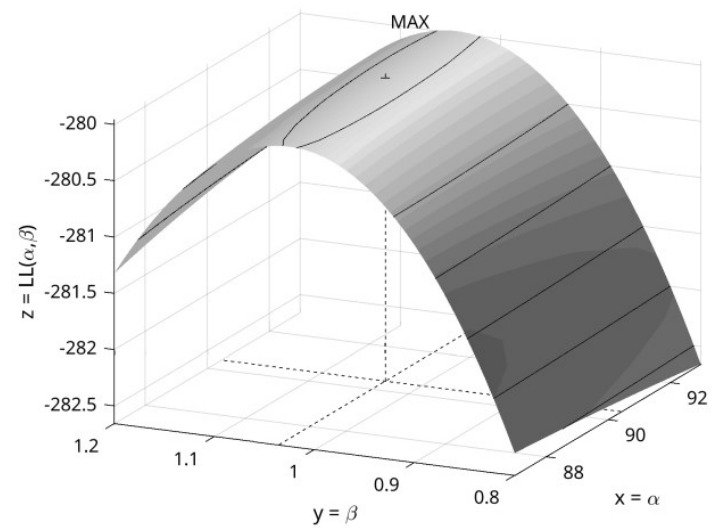

Figure 5: Android MLE approximation

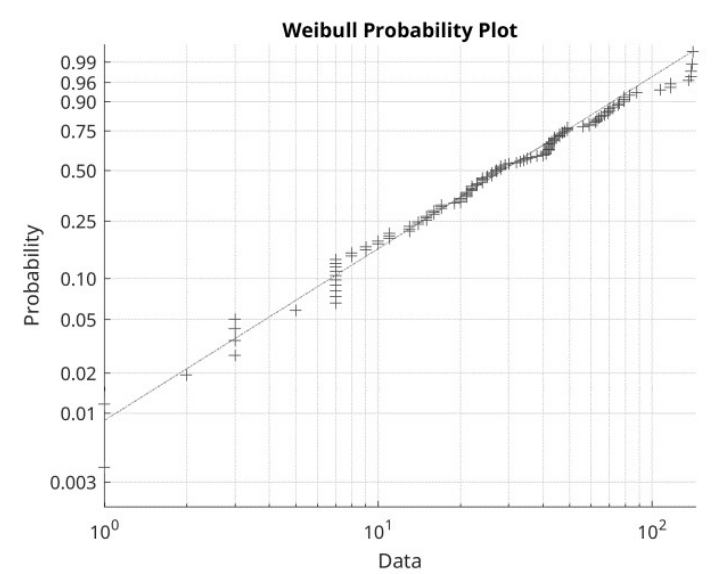

Figure 2: iOS Weibull probability plot

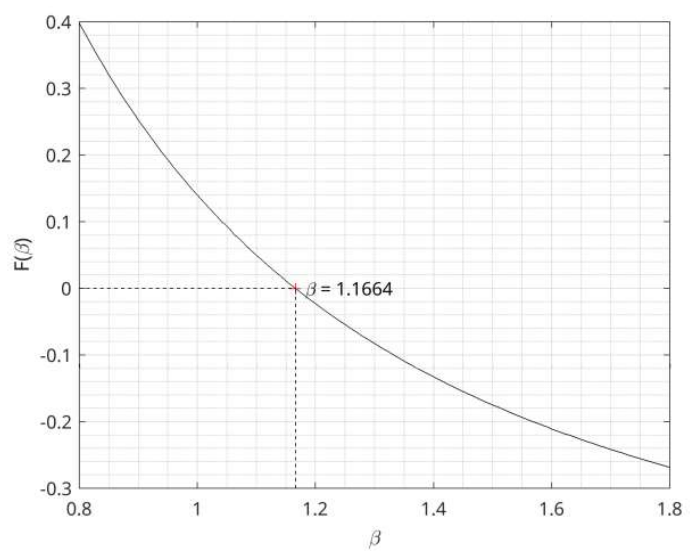

Figure 4: iOS Moments Method approximation

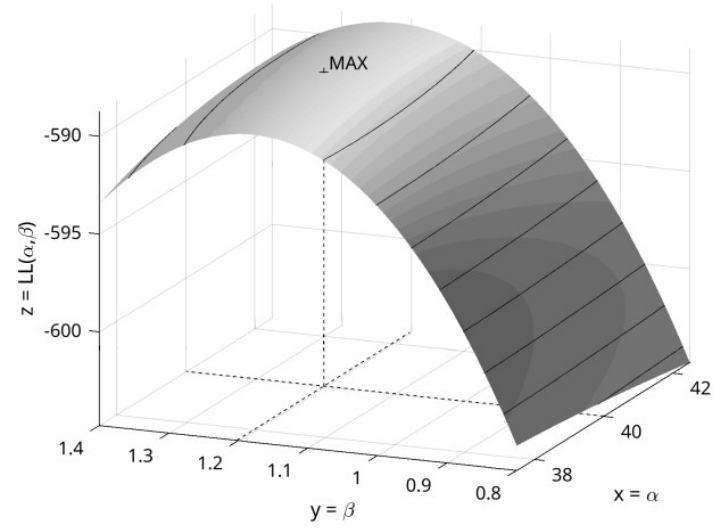

Figure 6: iOS MLE approximation 


\section{Goodness of fit}

The RMSE between the computed models and the collected life data are summarized in Table 4 for Android and Table 5 for iOS. RMSEs are expressed in days and different values are computed against different plotting positions.

Table 4: RMSE Android life data

\begin{tabular}{|l|l|l|l|l|l|l|l|}
\hline \multicolumn{1}{|c|}{ RMSE } & \multicolumn{1}{|c|}{ Position } & $\begin{array}{c}\text { Naïve } \\
\text { Estimator }\end{array}$ & \multicolumn{1}{|c|}{ Mean } & Midpoint & \multicolumn{1}{c|}{ Blom } & \multicolumn{1}{c|}{ Median } & \multicolumn{1}{c|}{ Mode } \\
\hline \multirow{4}{*}{ MOMENT } & & 0.0294 & 0.0279 & 0.0265 & 0.0267 & 0.0269 & 0.0261 \\
\hline \multirow{5}{*}{ REGRESSION } & Naïve Estimator & 0.0243 & 0.0263 & 0.0263 & 0.0262 & 0.0262 & 0.0275 \\
\cline { 2 - 8 } & Weibull \& Mean & 0.0270 & $\mathbf{0 . 0 2 3 7}$ & 0.0247 & 0.0243 & 0.0242 & 0.0271 \\
\cline { 2 - 9 } & Midpoint & 0.0284 & 0.0273 & 0.0259 & 0.0262 & 0.0263 & 0.0257 \\
\cline { 2 - 9 } & Blom & 0.0274 & 0.0257 & 0.0249 & 0.0250 & 0.0251 & 0.0254 \\
\cline { 2 - 9 } & Median & 0.0270 & 0.0250 & 0.0246 & 0.0246 & 0.0246 & 0.0255 \\
\hline \multirow{2}{*}{ MLE } & Mode & 0.0293 & 0.0290 & 0.0271 & 0.0275 & 0.0278 & 0.0263 \\
\hline
\end{tabular}

Table 5: RMSE iOS life data

\begin{tabular}{|c|c|c|c|c|c|c|c|}
\hline RMSE & Position & $\begin{array}{c}\text { Naïve } \\
\text { Estimator }\end{array}$ & Mean & Midpoint & Blom & Median & Mode \\
\hline MOMENT & & 0.0204 & 0.0194 & 0.0201 & 0.0199 & 0.0198 & 0.0209 \\
\hline \multirow{6}{*}{ REGRESSION } & Naïve Estimator & 0.0198 & 0.0195 & 0.0194 & 0.0194 & 0.0194 & 0.0195 \\
\hline & Weibull \& Mean & 0.0217 & 0.0204 & 0.0205 & 0.0204 & 0.0204 & 0.0208 \\
\hline & Midpoint & 0.0229 & 0.0222 & 0.0216 & 0.0218 & 0.0218 & 0.0213 \\
\hline & Blom & 0.0224 & 0.0215 & 0.0211 & 0.0212 & 0.0212 & 0.0210 \\
\hline & Median & 0.0222 & 0.0212 & 0.0209 & 0.0209 & 0.0210 & 0.0209 \\
\hline & Mode & 0.0234 & 0.0228 & 0.0221 & 0.0223 & 0.0224 & 0.0217 \\
\hline MLE & & 0.0218 & 0.0204 & 0.0206 & 0.0205 & 0.0205 & 0.0210 \\
\hline
\end{tabular}

\section{DISCUSSIONS}

From the goodness of fit, as shown by the results in Table 4 and Table 5, the minimum RMSE equals to 0.0244 days for Android and 0.0238 days for iOS. The best fits, for both models, correspond to the regression method, with the mean position for the Android, and to the Naïve Estimator position for the iOS. The corresponding Weibull couple of parameters are $\alpha=87.1794, \beta=0.9815$ for Android and $\alpha=41.9143, \beta=1.2709$ for iOS.

From the collected life data, the lifespan of an Android OS varies between 2 days and 393 days with a mean time between failures of 93.21 days, a large standard deviation of 96.67 days, a positive skewness of 2.11 and excess kurtosis of 6.77. Similarly, for the iOS, the shortest life is one day and the maximum is 141 days. The mean time between failures equals to 36.76 days and the standard deviation equals to 30.29 days. The skewness value of 1.48 and excess kurtosis of 
3.05 both indicate a right tail for the distribution. Both operating systems have a high standard deviation and, while Android detains the longest lifespan, iOS displays a lower dispersion. The PDF plotted in Figure 7 indicates the relative probability of failure at different times for both OS families. Both distributions are right-skewed, with Android having the larger right tail.

Since the failure rate is the time raised to the power of $(\beta-1)$, the value of the shape parameter, which is very close to unity for the Android life data, leads to the conclusion that the failure rate is almost constant over time as shown by the curve of the hazard rate function in Figure 8. For iOS family, however, the value of the shape parameter is greater than one, implying that the failure rate increases with time Figure 8. This can also be interpreted as the expression of an undergoing ageing or wearing out process, occurring to the OS as time advances.

$$
\begin{gathered}
h(x)=\frac{f(x)}{1-F(x)}=\left(\frac{\beta x^{\beta-1}}{\alpha^{\beta}}\right) \\
R(x)=e^{-\left(\frac{x}{\alpha}\right)^{\beta}}
\end{gathered}
$$

The instantaneous probability of failure per unit of time of equation 17 and plot in Figure 8 open the path to diverse computations such as: the instantaneous failure rate given a survival after one-year period ( $x=365$ days). The computations gave 1.00 and 5.08 per 100 days respectively for Android and iOS.

The proportion of the population expected to survive through time is expressed by the reliability function in equation 18 and displayed on by the survivorship curve in Figure 9. The probability for an OS version to fail before one month (30 days) are 29\% for Android and 51\% for iOS yielding the respective chance to survive during one-month period as $71 \%$ and $49 \%$. After three months' period (90 days), the probability for the Android OS to become obsolete is $63 \%$ and $94 \%$ for the iOS. The corresponding chances to survive are $37 \%$ and $6 \%$. After one-year period (360 days) though, there is no notable difference regarding the life performance of both OS since the corresponding probability of failure are $98 \%$ and $100 \%$ giving a survival chance of $2 \%$ and $0 \%$ respectively for Android and iOS.

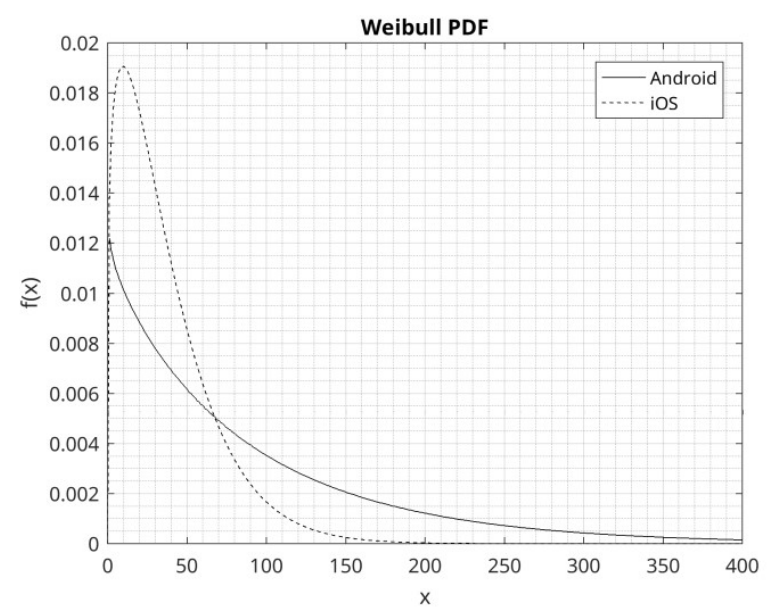

Figure 7: Weibull PDF plot from life data

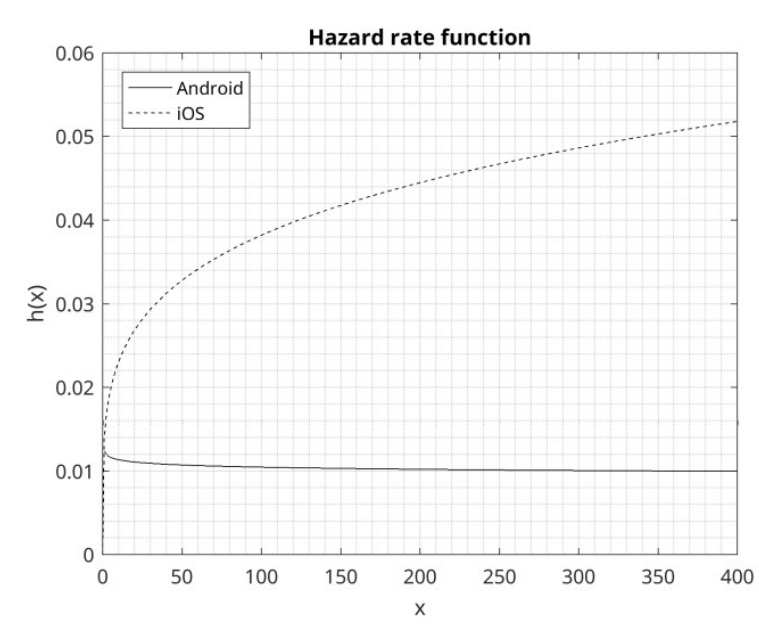

Figure 8: Hazard rate plot from life data 


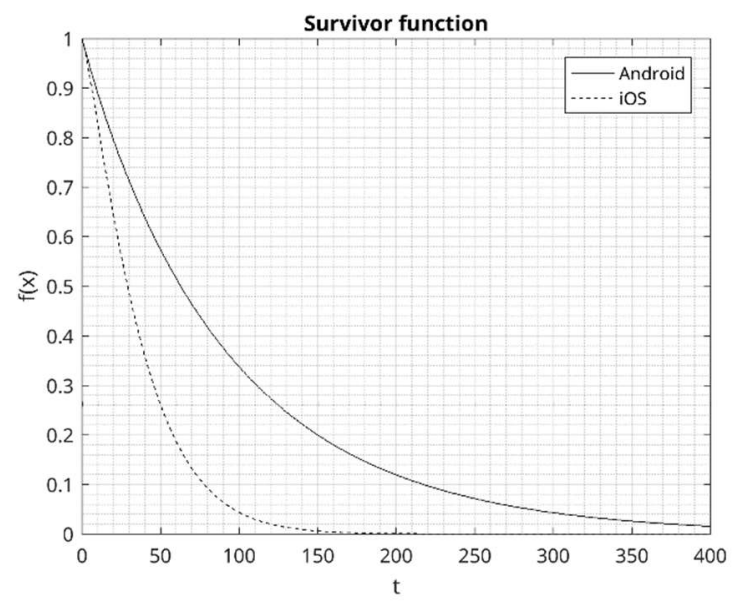

Figure 9: Survivor function curve

To end this discussion, it is worth mentioning that Android and iOS life data taken all together is not a twoparameter Weibull distribution. The null hypothesis from the Anderson-Darling test is rejected telling that there is not enough evidence supporting that the mixture follows a Weibull distribution. Detailed results are shown in Table 6.

Table 6: Parameters estimation from mixed data from Android and iOS

\begin{tabular}{|c|c|c|c|c|c|c|c|}
\hline \multirow[b]{2}{*}{ Method } & \multirow[b]{2}{*}{ Position } & \multicolumn{2}{|c|}{ Parameters } & \multicolumn{3}{|c|}{ Hypothesis test } & \multirow{2}{*}{ Test result } \\
\hline & & Shape $\beta$ & Scale $\alpha$ & AD & AD* & OSL & \\
\hline Moment & & 0.9044 & 49.7904 & 1.7289 & 1.7548 & 0.0002 & $H_{0}$ rejected \\
\hline \multirow{6}{*}{ Regression } & Naive Estimator & 1.1150 & 50.5379 & 1.0126 & 1.0278 & 0.0106 & $H_{0}$ rejected \\
\hline & Weibull \& Mean & 1.1003 & 51.6178 & 0.9904 & 1.0052 & 0.0120 & $H_{0}$ rejected \\
\hline & Midpoint & 1.1327 & 51.2831 & 1.1700 & 1.1875 & 0.0044 & $H_{0}$ rejected \\
\hline & Blom & 1.1235 & 51.3764 & 1.1101 & 1.1267 & 0.0061 & $H_{0}$ rejected \\
\hline & Median & 1.1184 & 51.4285 & 1.0801 & 1.0962 & 0.0072 & $H_{0}$ rejected \\
\hline & Mode & 1.1413 & 50.9216 & 1.2144 & 1.2326 & 0.0034 & $H_{0}$ rejected \\
\hline MLE & & 1.0108 & 52.5079 & 0.9374 & 0.9514 & 0.0163 & $H_{0}$ rejected \\
\hline
\end{tabular}

\section{CONCLUSIONS}

Mobile devices carry more and more sensitive personal and corporate data. Thus, carrying a secure mobile device became crucially important. In this inquiry, the usual approaches, which are aimed to finding the best way to educate the end-users about technological safety practices and accordingly adjusting behavior, have been put aside. Besides, the environment surrounding the making and the maintaining of a mobile operating system is a complex system, many parameters involved are including behavioral, corporate and financial factors. Simplifications have been made, in this study, to be able to focus on the main mobile operating system and the delivery pattern of updates and upgrades. The main expectation is to discover the underlying patterns matching the current delivery process and to draw a conclusion from those patterns.

The two-parameter-W( $\alpha, \beta)$ Weibull model has been used to model the life data of the two main mobile operating systems currently in use: the Android OS and the iOS. Three methods were used in order to build the model of mobile operating systems: the moment method, the regression method, and the maximum likelihood method. In order to check that 
the obtained models were valid, the Anderson-Darling hypothesis test was implemented and models were accepted or rejected according to the Observed Significance Level of the test. Finally, a goodness of fit test was carried out by computing the Root Mean Square Error of the models against the data.

Subsequent results show that the Android life data follow the Weibull law as: $\mathrm{W}(87.1794,0.9815)$ whereas the iOS life data follow the Weibull law as $\mathrm{W}(41.9143,1.2709)$. From the value of the shape parameter, Android life data exhibits a decreasing hazard rate, while the iOS life data shows an increasing hazard rate over time. The mean time to failure is around 93 days for Android and around 37 days for iOS. This study ends with a graphical plot of the hazard rate and the reliability function of the models in order to give a clear view and to draw the distinction between the two mobile OS.

Expectantly, this study opens the path and, at the same time, provides tools to a deeper study which encompasses technological alarm notification and human reaction. That being the case, the following questions require some demystifying answers: is there a border separating an alarm system from being dull to invasive? How to harmonize an alarm system to human behavior in order to be optimal?

\section{REFERENCES}

1. Swanepoel, "Smartphone-based National Hearing Test Launched in South Africa," The Hearing Journal: January -Volume 70-Issue 1, 2017, pp.14,16.

2. Sehgal K., Jain A., Nagrath P., Kumar A." Recent Advances in Networks and Data Security Survey on Various Mobile Operating Systems." In: Bhattacharyya S., Hassanien A., Gupta D., Khanna A., Pan I. (eds) International Conference on Innovative Computing and Communications. Lecture Notes in Networks and Systems, 2019 Vol 55. Springer, Singapore.

3. Android Open Source Project. https://source.android.com/ (Last visited on May 20, 2020).

4. Pavneet Singh Kochhar, Joseph Chan Joo Keng, Tan Biying, An Empirical Study on Bug Reports of Android 3rd Party Libraries Singapore Management University, Singapore: 2013.

5. Greene, D., \& Shilton, K, "Platform privacies: Governance, collaboration, and the different meanings of 'privacy' in iOS and Android development," New Media \& Society, 20(4), 2017, pp.1640-1657.

6. Jonathan Zittrain, The Future of the Internet - And How to Stop It Yale University Press, 2008.

7. Joyce, V. Jemmy, and K. REBBECA JEBASEELI EDNA. "Designing and Selection of Reliability Based Sampling Plans." International Journal of Mechanical and Production Engineering Research and Development (IJMPERD) 8.4 (2018): 343-348.

8. "Common Vulnerabilities and Exposures" https://cve.mitre.org (Last visit: May 20, 2020).

9. Mario Linares-Vásquez, Gabriele Bavota, Camilo Escobar-Velásquez, "An Empirical Study on Android-related Vulnerabilities." IEEE/ACM 14th International Conference on Mining Software Repositories (MSR), 2017.

10. Erik Derr, Sven Bugiel, Sascha Fahl, Yasemin Acar, Michael Backes, "Keep me Updated: An Empirical Study of Third-Party Library Updatability on Android," Proceedings of the 2017 ACM SIGSAC Conference on Computer and Communications Security, 2017, pp.2187-2200. 
11. Krishna, G. Sai, and G. T. N. Veerendra. "FLOOD FREQUENCY ANALYSIS OF PRAKASAM BARRAGE RESERVOIR KRISHNA DISTRICT, ANDHRA PRADESH USING WEIBULL, GRINGORTEN AND L-MOMENTS FORMULA." International Journal of Civil, Structural, Environmental and infrastructure Engineering Research and Development (IJCSEIERD) Vol 5.

12. Thomas, D. R., Beresford, A. R., \& Rice, “A. Security Metrics for the Android Ecosystem,” Proceedings of the 5th Annual ACM CCS Workshop on Security and Privacy in Smartphones and Mobile Devices, 2015, p.87-98.

13. I. Mohamed and D. Patel, “Android vs. iOS Security: A Comparative Study,” 2015 12th International Conference on Information Technology - New Generations, Las Vegas, NV, 2015, p 725-730.

14. Silas Formunyuy Verkijika, "Understanding smartphone security behaviors: An extension of the protection motivation theory with anticipated regret," Computers \& Security Volume 77, August 2018, pp.860-870.

15. Reddy, A. Chennakesava. "Studies on loading, cracking and clustering of particulates on the strength and stiffness of 7020/SiCp metal matrix composites." International Journal of Metallurgical \& Materials Science and Engineering 5.1 (2015): 53-66.

16. Bartlomie j Hanus \& Yu “Andy" Wu, "Impact of Users' Security Awareness on Desktop Security Behavior: A Protection Motivation Theory Perspective," Information Systems Management, 33:1, 2016, pp.2-16.

17. Alsaleh M, Alomar N, Alarifi A, "Smartphone users: Understanding how security mechanisms are perceived and new persuasive methods," PLoS ONE 12(3): e0173284, 2017.

18. Chitra, D., and P. Parvathi. "Decision making under fuzzy environment for deteriorating items with stock dependent demand under inflation effect." Int J Math Comput Appl Res 4.2 (2014): 1-10.

19. Murat Koyuncu and Tolga Pusatli, "Security Awareness Level of Smartphone Users: An Exploratory Case Study." Mobile Information Systems Volume 2019, 2019.

20. Tim Cooper, "Inadequate Life? Evidence of Consumer Attitudes to Product Obsolescence," Journal of Consumer Policy. December 2004, Volume 27, Issue 4, pp 421-449.

21. Volker G. Kuppelwieser, Phil Klaus, Aikaterini Manthiou, Othman Boujena, "Consumer responses to planned obsolescence," Journal of Retailing and Consumer Services Volume 47, 2019, pp.157-165

22. Naomi I. Eisenberger and Matthew D. Lieberman, "Why rejection hurts: a common neural alarm system for physical and social pain. TRENDS, " Cognitive Sciences Vol.8 No.7, July 2003.

23. Kees van den Bos, "Humans Making Sense of Alarming Conditions: Psychological Insight into the Fair Process Effect," The Oxford Handbook of Justice in the Workplace, Oxford University Press, 2015.

24. HORST Rinne, The Weibull Distribution: A Handbook, Taylor \& Francis Group, 2009.

25. John I. McCool, Using the Weibull Distribution: Reliability, Modeling, and Inference, John Wiley \& Sons, Inc., 2012.

26. Robert, Abernethy, The New Weibull Handbook. Fifth Edition, 2004.

27. Chin-Diew Lai, Generalized Weibull Distribution, Springer, 2014.

28. Nielsen Mark A, "Parameter Estimation for the Two-Parameter Weibull Distribution," All Theses and Dissertations Brigham Young University BYU Scholars Archive. 2509: 2011.

29. K. Krish Namoorthy, Handbook of Statistical Distributions with Applications, Taylor \& Francis Group, 2006.

30. D. N. Prabhakar Murthy, Min Xie, Renyan Jiang, Weibull Models, Wiley Series in Probability and Statistics, 2004. 
31. Sonja Engmann, Denis Cousineau, "Comparing Distributions: The Two-Sample Anderson-Darling Test As An Alternative To The Kolmogorov-Smirnoff Test," Journal of Applied Quantitative Methods Vol6 N3: 2011.

32. Jorge Luis Romeu, “Anderson-Darling: A Goodness of Fit Test for Small Samples Assumptions, " Reliability Analysis Center, START Volume 10, Number 5: 2003.

33. Rob J Hyndman, Anne B Koehler, “Another look at measures of forecast accuracy,” International Journal of Forecasting Volume 22, Issue 4, October-December 2006, Pages 679-688.

\section{AUTHOR PROFILE}

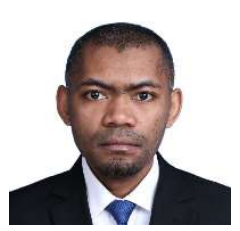

Julliard RALIHALIZARA received his Ph.D. in Complex Systems from Wuhan University of Technology, P.R. China. Having a solid background in engineering, with a focus on Finite Element Method (FEM), currently his main interests are mathematical modeling; complex systems and computer programming.

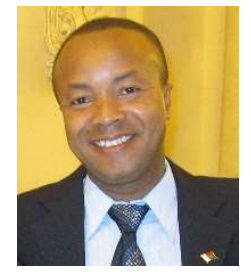

Jules Harris DANARSON received his Ph.D. in Administrative Management from Huazhong University of Science and Technology and is now an Associate Professor at the School of International Education, Guangxi University of Finance and Economics, P.R. China. His primary focus has been on communications and management of organizational behavior, but his research interests range from information management, community development, business negotiations to public administration and governance. 

